\title{
ACONTECIMIENTOS ESTRESANTES POR SEXO EN ESTUDIANTES \\ DEL PRIMER AÑO DE MEDICINA EN UNA ESCUELA PRIVADA
}

STRESSFUL EVENTS BY SEX IN FIRST YEAR MEDICAL STUDENTS IN A PRIVATE SCHOOL

*Miriam Sánchez Angeles, *Katya Esquivel Cañas

Campus Universitario Siglo XXI*

Correspondencia: miriamsanchezangeles@gmail.com

\section{RESUMEN}

El estrés es una respuesta ante diversos acontecimientos personales, familiares, económicos y académicos; que es experimentada por los individuos durante el transcurso de estos. El objetivo del trabajo consistió en medir la presencia, vigencia e intensidad de los sucesos estresantes en alumnos de medicina en una escuela privada y compararlos por sexo. Se estudiaron 128 alumnos a los que se les aplicó la Escala de Apreciación del Estrés (EAE) en su versión general, desarrollada por Fernández y Mielgo. Con los datos se realizó una t de Student en cada una de las categorías que evalúa el instrumento y se encontraron diferencias significativas en la intensidad y la vigencia (actual) de los eventos estresantes, siendo mayor la media de las mujeres que la de los hombres. En el caso de la presencia, la intensidad y la vigencia pasada no se encontraron diferencias estadísticamente significativas.

Palabras clave: estudiantes de medicina, eventos estresantes, educación médica.

\section{ABSTRACT}

The stress as a response to different events that goes from personal, familiar, economical and academic is commonly experience by individuals during the 
lifespan. The purpose of the investigation was to detect the presence, impact and intensity of stressful events in freshmen of medicine in a private school and compare them by sex. The study included a total of 128 students that answered the Stress Appreciation Scale in its General Form (SAS-G) of Fernandez and Mielgo. A $t$ of Student by sex was conducted in each of the categories included in the instrument; the differences were found in Intensity (Present) and Validity (Present) of the stressful events; being the women's average superior to men. The Presence, Intensity and Validity in the past were not significant in terms of statistics.

Key words: Medical students, Stressful events, Medical education.

\section{INTRODUCCIÓN}

Actualmente, es común escuchar en conversaciones entre pares académicos e incluso entre jóvenes universitarios la palabra estrés, misma que es utilizada de manera indiscriminada para definir una serie de signos y síntomas que alteran la salud tanto física como psicológica. Si bien estas nociones tienen cierto grado de certeza, es necesario aclarar algunos aspectos fundamentales sobre este tema con el propósito de resaltar la importancia de estudiarlo en el área de la salud especialmente en la carrera de medicina.

De acuerdo con la American Psychological Association (2019) el estrés es una reacción a una situación de corta duración; por ejemplo, estar en un atasco en el tráfico o una respuesta de larga duración, que puede presentarse al experimentar la muerte de un familiar cercano. La APA reconoce que el estrés se convierte en algo peligroso cuando interfiere con la habilidad para vivir (relativamente normal) durante un periodo de tiempo prolongado, ya que el estrés puede generar daños a nivel físico.

En este sentido, el estrés puede ser entendido como un estímulo externo que conduce a la acción, misma que en muchos casos es interpretada como una respuesta vital para la supervivencia. Sin embargo, las respuestas no siempre son coherentes con el entorno, ya que éste puede involucra respuestas insuficientes o exageradas por parte del individuo. 
En consonancia con los autores del instrumento, el estrés es "una valoración cognitiva y afectivo-emocional que le da el sujeto a los diferentes estímulos, agentes, circunstancias, situaciones y factores que amenazan la vida del sujeto, su salud física y psíquica" (Fernández \& Mielgo, 2001).

El estrés está asociado a diversos problemas físicos y psicológicos; de acuerdo con la OMS de los 75 mil infartos registrado al año en México un 25\% se asocian al estrés laboral. Este mismo organismo indica que el caso de México es relevante pues el $75 \%$ de los trabajadores lo padece y su porcentaje es incluso mayor que en China (73\%) lugar en el cual los suicidios son frecuentemente asociados a este mal (Forbes, 21 de diciembre de 2017).

Si bien el ritmo acelerado de vida afecta a la mayoría de la población, se pueden identificar algunos grupos o sujetos más vulnerables a estos fenómenos, las posibilidades de que un conjunto de sujetos se vea mayormente expuesto a vivenciar dichos estados se debe a características como la ocupación, el nivel socioeconómico, las actividades diarias, las responsabilidades, incluso la zona de residencia y el nivel de estudios entre otros factores.

Aun cuando estos datos hacen referencia al sector laboral en México, esta situación puede parecer ajena al grupo de estudio del presente trabajo; pero no se debe obviar que las estadísticas de suicidio en médicos residentes en el país los colocan en un grupo vulnerable a este problema. De acuerdo con Becerril (19 de agosto de 2018) la Comisión Permanente remitió un documento a la Secretaria de Salud en donde alerta sobre la falta de estudios sobre esta materia y hacen notar que de estos profesionistas alrededor de 300 a 400 médicos se quitan la vida cada año, es decir, en promedio uno al día. Además, se menciona que los casos de suicidio o intentos aumentan en los periodos de ingreso a las escuelas de medicina, al inicio de la residencia y en los médicos cuando se enfrentan a la jubilación.

En este sentido en las licenciaturas pertenecientes al área de la salud se puede observar que las exigencias estudiantiles son de alto impacto, ya que el manejo del estado de salud física y emocional requiere una capacitación constante, así como precisión en los procesos de intervención, diagnóstico y estudio. En la investigación titulada El bienestar de los Estudiantes realizado por la OCDE (Ministerio de Educación, Cultura y Deporte, 2015) se encontró que la mitad de los jóvenes mexicanos que se encuentran estudiando experimentan ansiedad y estrés respecto a su desempeño académico. 
El contar con instrumentos que ofrezcan datos confiables sobre este problema contribuye no solamente al diagnóstico de un posible riesgo de salud, sino que también contribuye al análisis del bienestar psicológico, que es "un constructo que incluye el sentir positivo y pensar del ser humano acerca de sí mismo, y que se relaciona con aspectos como el funcionamiento psíquico, físico y social" (GarcíaViniegras \& González, 2000).

El retomar un instrumento como la Escala General de Estrés EAE-G, que aporta datos como la presencia de sucesos estresantes, la vigencia actual o pasada de dicho evento y la intensidad con la que el sujeto experimentó o sigue experimentando, abre un panorama amplio para potenciar el bienestar psicológico en la población a la cual se dirige la presente investigación.

Aunado a ello, para poder discriminar de manera más sensible los datos, se hace una distinción por sexo entendido como las características físicas, biológicas, anatómicas y cromosómicas que distinguen entre hombre o mujer. En el caso del presente estudio se retoma esta diferenciación dado que los mismos sucesos estresantes pueden ser vividos de manera diferente de acuerdo con los roles y estereotipos propios, tener en consideración esta diferencia puede ayudar a hacer las sugerencias pertinentes para visibilizar el contraste de apreciaciones frente al estrés.

En este trabajo se considera que analizar esta categoría conceptual desde una perspectiva de género enriquece el entendimiento de este fenómeno y abona a la necesidad de reflexionar sobre los estudios psicológicos desde una visión social.

\section{MÉTODO}

Se realizó un estudio cuyo objetivo consistió en explorar la presencia, vigencia e intensidad de los eventos estresantes experimentados por los alumnos inscritos al primer año de la licenciatura en medicina de una institución de educación superior para analizar las diferencias entre estos eventos por sexo. 


\section{Participantes}

La población de estudio se conformó por la totalidad de alumnos inscritos al primer año de la carrera de medicina de una escuela de régimen privado ubicada en el municipio de Zinacantepec. De acuerdo con los datos del departamento de Control Escolar para ese ciclo se tenía registro de 149 alumnos inscritos, de los cuales se lograron recolectar 128 instrumentos válidos, obteniendo una cobertura del 85.9\% en el grupo de estudio.

Para la aplicación se contó con el apoyo de la coordinadora del área de ciencias básicas que autorizó las visitas a las aulas para dar respuesta al instrumento. La encargada de la aplicación fue una estudiante del último semestre de la licenciatura en psicología previamente capacitada en un curso de metodología de la investigación. Antes de la aplicación del instrumento se informó a los alumnos el objetivo del estudio, el uso que se haría de la información proporcionada y se les aseguró que su identidad se mantendría en el anonimato. En todo momento se comunicó que aquellos alumnos que no quisieran contestar el instrumento estaban en la libertad de declinar su participación, en caso de que esto ocurriera los alumnos no recibían formato del instrumento. Cabe aclarar que los estudiantes no recibieron compensación económica o de otro tipo por su participación en la investigación.

La distribución por género fue de $62.5 \%$ femenino y $37.5 \%$ masculino. La edad de los alumnos al momento de la aplicación osciló entre los 18 y 31 años $(M=19.13$, $D E=1.70)$.

\section{Instrumento}

Se eligieron las Escalas de Apreciación del Estrés (EAE) publicada por TEA Editores y desarrolladas por José Luis Fernández Seara y Manuela Mielgo Robles. El instrumento se compone de cuatro escalas diferentes:

I EAE-G: Escala General de Estrés

1 EAE-A: Escala de Acontecimientos Estresantes en Ancianos

I EAE-S: Escala de Estrés Socio-Laboral

I EAE-C: Escala de Estrés en la Conducción 
Todas las escalas tienen el objetivo de analizar la incidencia y el peso de los distintos acontecimientos en la vida de los sujetos. Sin embargo, las últimas tres (A, $\mathrm{S}$ y C) se descartaron pues no se relacionan con las características de la población de estudio.

En el caso de la EAE-G (la elegida para este estudio) se incluyen reactivos que valoran la percepción de la vida académica que es uno de los aspectos que interesaba en este trabajo. El instrumento va dirigido a la población en general abarcando edades entre los 18 y los 60 años. Una de las variables de control es la edad y el sexo. Esta escala en particular consta de 53 elementos, el instrumento cuenta con una confiabilidad test-retest de 0.65 y una confiabilidad por mitades de 0.74 .

El desarrollo del instrumento tiene como modelos teóricos y líneas de investigación, las desarrolladas por Holmes y Rahe (1967) y Lazarus (1966). Se considera que, a lo largo del ciclo vital de la persona, se dan actividades, desempeño de roles, vivencias de sucesos, experiencias, situaciones que desencadenan estrés, ansiedad, nerviosismo, tensión y otros síntomas en los sujetos. No obstante, no todos los agentes potencialmente estresantes están presentes en la vida de cada individuo, ni afectan de la misma manera. Al contrario, la respuesta al estrés es diferencial, depende de los agentes pero también de la reacción y capacidad de afrontamiento por parte del individuo.

\section{Procedimiento}

En sintonía con el objetivo de este estudio, no se realizaron modificaciones ni adaptaciones del instrumento original. En la base de datos que se diseñó para la captura y procesamiento se identificó a los participantes con un folio ascendente sin duplicados. Después de la aplicación se revisaron todas las hojas de respuesta, desechando aquellas que presentaban respuestas inválidas (3 casos), ya sea por omisión o porque seleccionaron más de una casilla en cualquiera de las respuestas.

Para calificar el instrumento se analizan tres categorías en el siguiente orden:

1 Presencia: Pretende conocer el número de sucesos estresantes que han ocurrido en la vida del sujeto. 
Esta es una variable discreta, medida en el nivel ordinal con dos categorías, Presencia (SI) o Ausencia (NO) del acontecimiento estresante en la vida del sujeto. El resultado oscila entre 0 y 53 pues sólo mide la frecuencia.

1 Vigencia del acontecimiento estresante: Analiza la discriminación de los agentes estresantes en el tiempo, es decir, si los acontecimientos estresantes han dejado de afectarle o todavía le siguen afectando.

Esta es una variable discreta, medida en el nivel ordinal con dos categorías

Si ha dejado de afectarle Pasado $(P)$ o si todavía le afecta Actual $(A)$. El resultado oscila entre 0 y 53 pues solamente se reporta la frecuencia en cada reactivo.

I Intensidad: Busca apreciar una valoración personal del grado con que cada uno vive o ha vivido los sucesos estresantes.

Esta es una variable continua, medida a nivel ordinal en cuatro rangos (0 123 ). Cabe aclarar que esta escala se une con la de vigencia, es decir, se mide la intensidad de los eventos Pasados y Actuales. El puntaje se mueve entre O y 159 puntos en ambos casos.

\section{Consideraciones éticas}

En cada una de las aplicaciones se incluyó como primera página el consentimiento informado. Los alumnos que eligieron participar en el estudio, respondieron de manera voluntaria y firmaron dicho documento con el objetivo de poder contar con evidencia de su aprobación para ser incluidos en el estudio, así como la autorización de la futura publicación de los resultados grupales. El aplicador dio las instrucciones precisas para responder al cuestionario asegurándoles que no existían respuestas buenas ni malas por lo que les pidió que contestaran con la mayor sinceridad posible.

\section{RESULTADOS}

Para poder explorar el instrumento, en la Tabla 1 se presentan los estadísticos descriptivos por sexo, así como de la totalidad de alumnos. 
Tabla 1. Estadísticos descriptivos

\begin{tabular}{|c|c|c|c|c|c|c|c|c|}
\hline & & Categoría & $\mathrm{N}$ & Min & $\operatorname{Max}$ & $\mathrm{Me}$ & $M$ & $\mathrm{De}$ \\
\hline \multirow{3}{*}{\multicolumn{2}{|c|}{ 1. Presencia }} & Hombre & 48 & 3 & 28 & 14 & 13.8 & 5.7 \\
\hline & & Mujer & 80 & 5 & 37 & 16 & 15.9 & 7.0 \\
\hline & & Población & 128 & 3 & 37 & 15 & 15.2 & 6.6 \\
\hline \multirow{6}{*}{ 2. Vigencia } & \multirow{4}{*}{ Actual } & Hombre & 48 & 0 & 22 & 8 & 8.3 & 4.8 \\
\hline & & Mujer & 80 & 1 & 30 & 11 & 10.8 & 5.3 \\
\hline & & Población & 128 & 0 & 30 & 9 & 9.9 & 5.3 \\
\hline & & Hombre & 48 & 0 & 16 & 5 & 5.4 & 3.7 \\
\hline & \multirow[t]{2}{*}{ Pasado } & Mujer & 80 & 0 & 25 & 5 & 5.1 & 4.9 \\
\hline & & Población & 128 & 0 & 25 & 5 & 5.3 & 4.5 \\
\hline \multirow{6}{*}{ 3. Intensidad } & \multirow{3}{*}{ Actual } & Hombre & 48 & 0 & 42 & 14 & 14.9 & 10.3 \\
\hline & & Mujer & 80 & 2 & 87 & 20 & 22.4 & 13.7 \\
\hline & & Población & 128 & 0 & 87 & 17 & 19.6 & 13.0 \\
\hline & \multirow{3}{*}{ Pasado } & Hombre & 48 & 0 & 23 & 6 & 7.6 & 6.1 \\
\hline & & Mujer & 80 & 0 & 50 & 6 & 9.4 & 10.4 \\
\hline & & Población & 128 & 0 & 50 & 6 & 8.8 & 9.0 \\
\hline
\end{tabular}

Fuente: Elaboración propia a partir de la base de datos

Como es posible apreciar, los estudiantes de primer año de medicina reconocen haber experimentado en promedio 15 sucesos estresantes (28\%) de los 53 que explora el instrumento. Existe una media mayor de sucesos estresantes que aún les siguen afectando (9.9) a diferencia de los que han dejado de afectarles (5.3). La intensidad reportada es mayor en aquellos eventos experimentados en la actualidad (19.6) que en aquellos del pasado (8.8).

Finalmente, respecto a la comparación por sexo, en la gráfica 1 se muestran los promedios de cada una de las categorías exploradas por el instrumento.

Gráfica 1. Promedios por sexo

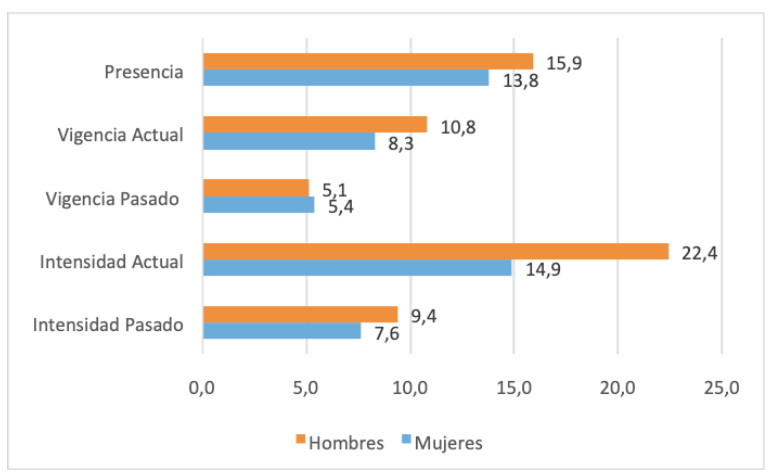

Fuente: Elaboración propia a partir de la base de datos 
Lo primero que resalta en la gráfica es que la percepción que tienen las mujeres ante los eventos estresantes es mayor a la de los hombres en 4 de las 5 categorías que pudieron explorarse. Este hecho es notable y debe considerarse para la futura discusión de los resultados.

No obstante, para poder determinar si dicha diferencia es estadísticamente significativa se requiere realizar una prueba de comparación, dado que las tres categorías miden sus variables a nivel ordinal, la prueba estadística elegida es la $t$ de Student para muestras independientes. Teniendo presente estas diferencias pasemos a analizar las implicaciones teóricas de este trabajo en términos del instrumento, cabe notar que en cada una de las categorías se incluyen los reactivos con las frecuencias más elevadas por género.

\section{Presencia}

Es una categoría que explora los acontecimientos estresantes y se refiere a ellos como estímulos, agentes, circunstancias, situaciones o factores que amenazan la vida, la salud psíquica y la salud mental.

En primer lugar, respecto a la comparación por sexo, las diferencias no fueron estadísticamente significativas entre hombres $(M=13.81, D E=5.7)$ y mujeres $(M=15.96$, $D E=7.0$ ) pues se obtuvo una $\mathrm{t}(126)=-1.80, \mathrm{p}=0.073$.

Tabla 2. $t$ de Student: Presencia

\begin{tabular}{|c|c|c|c|c|c|c|c|c|c|c|}
\hline \multicolumn{11}{|c|}{ Prueba de muestras independientes } \\
\hline & & \multicolumn{2}{|c|}{$\begin{array}{l}\text { Prueba de Levene de } \\
\text { igualdad de varianzas }\end{array}$} & \multicolumn{7}{|c|}{ prueba t para la igualdad de medias } \\
\hline & & & & & & & & Diferencia de & $\begin{array}{l}95 \% \text { de in } \\
\text { confianza de }\end{array}$ & $\begin{array}{l}\text { alo de } \\
\text { diferencia }\end{array}$ \\
\hline & & $\mathrm{F}$ & Sig. & $\mathrm{t}$ & gl & (bilateral) & $\begin{array}{l}\text { Dlierencla ae } \\
\text { medias }\end{array}$ & estándar & Inferior & Superior \\
\hline \multirow[t]{2}{*}{ Presencia } & $\begin{array}{l}\text { Se asumen varianzas } \\
\text { iguales }\end{array}$ & 1,554 & 215 & $-1,805$ & 126 & ,073 & $-2,150$ & 1,191 & $-4,507$ & ,207 \\
\hline & $\begin{array}{l}\text { No se asumen varianzas } \\
\text { iguales }\end{array}$ & & & $-1,899$ & 114,621 &, 060 & $-2,150$ & 1,132 & $-4,393$ & 093 \\
\hline
\end{tabular}

Fuente: Base de datos SPSS

En segundo lugar, respecto a las frecuencias simples, las mujeres (8) han experimentado más sucesos que los hombres (5). Ahora bien, ¿cuáles fueron estos sucesos?, los datos pueden verificarse en la tabla 3. 
Tabla 3. Frecuencia de reactivos: Presencia

\begin{tabular}{|lcc|}
\hline \multicolumn{1}{c}{ Hombres (Total:48) } & Frec & $\%$ \\
\hline R03. Época de exámenes o exposiciones & 43 & 89.6 \\
\hline R16. Preocupación por la puntualidad & 34 & 70.8 \\
\hline R13. Hablar en público & 32 & 66.7 \\
\hline R01. Primer empleo & 29 & 60.4 \\
\hline R47. Éxito o fracaso profesional & 25 & 52.1 \\
\hline \multicolumn{1}{c|}{ Mujeres (Total:80) } & Frec & $\%$ \\
\hline R03. Época de exámenes o exposiciones & 77 & 87.5 \\
\hline R19. Presencia de una persona no deseada en el hogar (tía, abuela, pariente) & 63 & 78.8 \\
\hline R13. Hablar en público & 55 & 68.8 \\
\hline R41. Exceso de trabajo & 53 & 66.3 \\
\hline R47. Éxito o fracaso profesional & 51 & 63.8 \\
\hline R08. Estilo de vida y/o forma de ser & 49 & 61.3 \\
\hline R07. Problemas económicos propios o familiares & 48 & 60.0 \\
\hline R11. Falta de apoyo o traición de los amigos & 43 & 53.8 \\
\hline
\end{tabular}

Fuente: Elaboración propia a partir de la base de datos

Resalta que más del $85 \%$ de mujeres y hombres han experimentado como estresante la época de exámenes o exposiciones. Esto resulta consonante con los trabajos de Rodríguez, Sanmiguel, Muñoz y Rodríguez (2014) que destacan que el área médica se caracteriza por presentar una constante y creciente exigencia académica, que demanda al estudiante enormes esfuerzos de adaptación, por lo que resulta un ambiente lleno de elementos potenciadores de estrés.

De la misma forma, Segura (2015) encontró que al medir el estrés en estudiantes de medicina al inicio y final de su carrera se identificaron como factores estresantes: el grado académico, el enfrentarse al ejercicio medico en ámbitos reales, la complejidad de los contenidos y el aprovechamiento académico.

Respecto a los sucesos estresantes que aquí se analizaron, se observa que las mujeres reportaron: la presencia de una persona no deseada en el hogar (R19), el exceso de trabajo (R41), estilo de vida (R08), y problemas económicos propios o familiares (RO7) mismos que se relacionan con el ámbito familiar y laboral, esto puede apuntar a interpretar que la exigencia hacia las mujeres para desempeñarse de manera óptima en diferentes roles y espacios (público/privado) puede considerarse como causa de un mayor desgaste físico y emocional. Esta observación puede 
confirmarse con los resultados de los hombres que sólo manifestaron estrés por sucesos experimentados en la vida pública, espacio al que históricamente se les ha socializado para desempeñarse.

\section{Vigencia.}

Explora si el evento estresante es algo que continúa afectándole o ya ha dejado de hacerlo. En el caso de estas comparaciones, existió una diferencia significativa en la vigencia actual entre hombres $(M=8.38, D E=4.8)$ y mujeres $(M=10.85, D E=5.3)$ siendo menor la media de los hombres $\mathrm{t}(126)=-2.63, p=0.010$.

En los eventos del pasado no existieron diferencias significativas entre hombres $(M=5.46, D E=3.7)$ y mujeres $(M=5.18, D E=4.9)$ con $\mathrm{t}(126)=0.34, p=0.731$.

Tabla 4. $t$ de Student: Vigencia

\begin{tabular}{|c|c|c|c|c|c|c|c|c|c|c|}
\hline \multicolumn{11}{|c|}{ Prueba de muestras independientes } \\
\hline & & \multicolumn{2}{|c|}{$\begin{array}{l}\text { Prueba de Levene de } \\
\text { igualdad de varianzas }\end{array}$} & \multicolumn{7}{|c|}{ prueba t para la igualdad de medias } \\
\hline & & \multirow[b]{2}{*}{$\mathrm{F}$} & \multirow[b]{2}{*}{ Sig. } & \multirow[b]{2}{*}{$\mathrm{t}$} & \multirow[b]{2}{*}{$\mathrm{gl}$} & \multirow{2}{*}{$\begin{array}{c}\text { Sig. } \\
\text { (bilateral) }\end{array}$} & \multirow{2}{*}{$\begin{array}{l}\text { Diferencia de } \\
\text { medias }\end{array}$} & \multirow{2}{*}{$\begin{array}{c}\text { Diferencia de } \\
\text { error } \\
\text { estándar }\end{array}$} & \multicolumn{2}{|c|}{$\begin{array}{l}95 \% \text { de intervalo de } \\
\text { confianza de la diferencia }\end{array}$} \\
\hline & & & & & & & & & Inferior & Superior \\
\hline \multirow[t]{2}{*}{ V. Actual } & $\begin{array}{l}\text { Se asumen varianzas } \\
\text { iguales }\end{array}$ & ,605 &, 438 & $-2,631$ & 126 & 010 & $-2,475$ & 941 & $-4,337$ &,- 613 \\
\hline & $\begin{array}{l}\text { No se asumen varianzas } \\
\text { iguales }\end{array}$ & & & $-2,697$ & 106,879 &, 008 & $-2,475$ & 918 & $-4,294$ &,- 656 \\
\hline \multirow[t]{2}{*}{ V. Pasado } & $\begin{array}{l}\text { Se asumen varianzas } \\
\text { iguales }\end{array}$ & 1,057 & ,306 & ,344 & 126 &, 731 & ,283 &, 824 & $-1,347$ & 1,914 \\
\hline & $\begin{array}{l}\text { No se asumen varianzas } \\
\text { iguales }\end{array}$ & & &, 368 & 118,759 &, 714 &, 283 &, 771 & $-1,243$ & 1,809 \\
\hline
\end{tabular}

Fuente: Base de datos SPSS

La tabla 5 muestra el comportamiento de los reactivos. Como puede observarse un $90 \%$ de mujeres y un $75 \%$ de los hombres reportaron sentir estrés a causa de la época de exámenes o exposiciones. En segundo lugar, ambos géneros indican la puntualidad como una preocupación vigente. 
Tabla 5. Frecuencia de reactivos: Vigencia

\begin{tabular}{|l|c|c|}
\hline \multicolumn{1}{|c|}{ Hombres (Total:48) } & Frec & $\%$ \\
\hline R03. Época de exámenes o exposiciones & 43 & 89.6 \\
\hline R16. Preocupación por la puntualidad & 34 & 70.8 \\
\hline R13. Hablar en público & 32 & 66.7 \\
\hline R01. Primer empleo & 29 & 60.4 \\
\hline R47. Éxito o fracaso profesional & 25 & 52.1 \\
\hline \multicolumn{1}{|c|}{ Mujeres (Total:80) } & Frec & $\%$ \\
\hline R03. Época de exámenes o exposiciones & 77 & 87.5 \\
\hline R19. Presencia de una persona no deseada en el hogar (tía, abuela, pariente) & 63 & 78.8 \\
\hline R13. Hablar en público & 55 & 68.8 \\
\hline R41. Exceso de trabajo & 53 & 66.3 \\
\hline R47. Éxito o fracaso profesional & 51 & 63.8 \\
\hline R08. Estilo de vida y/o forma de ser & 49 & 61.3 \\
\hline R07. Problemas económicos propios o familiares & 48 & 60.0 \\
\hline R11. Falta de apoyo o traición de los amigos & 43 & 53.8 \\
\hline
\end{tabular}

Fuente: Elaboración propia a partir de la base de datos

Resulta interesante que las mujeres no reportaron frecuencias elevadas en sucesos estresantes del pasado, aquí vemos un área de oportunidad para medir resiliencia en estas poblaciones.

\section{Intensidad.}

Es una categoría que explora la valoración personal de la fuerza con que le han afectado los acontecimientos estresantes, esta categoría distingue entre acontecimientos de la actualidad y del pasado.

En la comparación por género, existieron diferencias significativas en los eventos actuales entre hombres $(M=14.98, D E=10.3)$ y mujeres $(M=22.43, D E=13.7)$ siendo menor la intensidad percibida por los hombres $\mathrm{t}(126)=-3.24, p=0.001$. En contraste, los eventos estresantes en el pasado no tuvieron diferencias estadísticas entre hombres $(M=7.65, D E=6.1)$ y mujeres $(M=9.49, D E=10.4)$ resultando una $\mathrm{t}(126)=-1.11$, $p=0.266$. 
Tabla 6. $t$ de Student: Intensidad

\begin{tabular}{|c|c|c|c|c|c|c|c|c|c|c|}
\hline \multicolumn{11}{|c|}{ Prueba de muestras independientes } \\
\hline & & \multicolumn{2}{|c|}{$\begin{array}{l}\text { Prueba de Levene de } \\
\text { igualdad de varianzas }\end{array}$} & \multicolumn{7}{|c|}{ prueba t para la igualdad de medias } \\
\hline & & \multirow[b]{2}{*}{$\mathrm{F}$} & \multirow[b]{2}{*}{ Sig. } & \multirow[b]{2}{*}{$\mathrm{t}$} & \multirow[b]{2}{*}{$\mathrm{gl}$} & \multirow[b]{2}{*}{$\begin{array}{c}\text { Sig. } \\
\text { (bilateral) }\end{array}$} & \multirow[b]{2}{*}{$\begin{array}{l}\text { Diferencia de } \\
\text { medias }\end{array}$} & \multirow{2}{*}{$\begin{array}{l}\text { Diferencia de } \\
\text { error } \\
\text { estándar }\end{array}$} & \multicolumn{2}{|c|}{$\begin{array}{l}95 \% \text { de intervalo de } \\
\text { confianza de la diferencia }\end{array}$} \\
\hline & & & & & & & & & Inferior & Superior \\
\hline \multirow[t]{2}{*}{ I. Actual } & $\begin{array}{l}\text { Se asumen varianzas } \\
\text { iguales }\end{array}$ & 2,281 & ,133 & $-3,247$ & 126 & ,001 & $-7,446$ & 2,293 & $-11,984$ & $-2,908$ \\
\hline & $\begin{array}{l}\text { No se asumen varianzas } \\
\text { iguales }\end{array}$ & & & $-3,482$ & 119,575 &, 001 & $-7,446$ & 2,138 & $-11,679$ & $-3,212$ \\
\hline \multirow[t]{2}{*}{ I. Pasado } & $\begin{array}{l}\text { Se asumen varianzas } \\
\text { iguales }\end{array}$ & 5,242 &, 024 & $-1,117$ & 126 & ,266 & $-1,842$ & 1,649 & $-5,105$ & 1,422 \\
\hline & $\begin{array}{l}\text { No se asumen varianzas } \\
\text { iguales }\end{array}$ & & & $-1,262$ & 125,981 & ,209 & $-1,842$ & 1,459 & $-4,729$ & 1,046 \\
\hline
\end{tabular}

Fuente: Base de datos SPSS

La tabla 7 incluye los reactivos con la mayor intensidad por sexo. En esta última categoría, se puede observar que hombres y mujeres en general valoran con intensidad la época de exámenes. No obstante, son las mujeres las que dan a este suceso un mayor puntaje. En contraste, el evento estresante experimentado en el pasado y valorado con mayor intensidad fue la muerte de algún familiar muy cercano.

Tabla 7. Frecuencia de reactivos: Intensidad

\begin{tabular}{|c|c|c|c|c|c|}
\hline \multicolumn{6}{|c|}{ Hombres (Total:48) } \\
\hline Actual & $\#$ & $\%$ & Pasado & $\#$ & $\%$ \\
\hline $\begin{array}{l}\text { R03. Época de exámenes o } \\
\text { exposiciones }\end{array}$ & 17 & 35.4 & $\begin{array}{l}\text { R28. Muerte de algún familiar muy } \\
\text { cercano }\end{array}$ & 4 & 8.3 \\
\hline R47. Éxito o fracaso profesional & 9 & 18.8 & $\begin{array}{l}\text { R21. Ruptura de noviazgo o } \\
\text { separación de pareja }\end{array}$ & 3 & 6.3 \\
\hline $\begin{array}{l}\text { R16. Preocupación por la } \\
\text { puntualidad }\end{array}$ & 7 & 14.6 & $\begin{array}{l}\text { R29. Muerte de algún amigo/a o } \\
\text { persona querida }\end{array}$ & 3 & 6.3 \\
\hline R14. Inseguridad ciudadana & 6 & 12.5 & R01. Primer empleo & 2 & 4.2 \\
\hline R34. Enfermedad de un ser querido & 6 & 12.5 & $\begin{array}{l}\text { R30. Embarazo no deseado o } \\
\text { deseado con problemas }\end{array}$ & 2 & 4.2 \\
\hline \multicolumn{6}{|l|}{ Mujeres (Total:80) } \\
\hline Actual & $\#$ & $\%$ & Pasado & $\#$ & $\%$ \\
\hline $\begin{array}{l}\text { R03. Época de exámenes o } \\
\text { exposiciones }\end{array}$ & 46 & 57.5 & $\begin{array}{l}\text { R28. Muerte de algún familiar muy } \\
\text { cercano }\end{array}$ & 11 & 13.8 \\
\hline R41. Exceso de trabajo & 25 & 31.2 & R34. Enfermedad de un ser querido & 9 & 11.3 \\
\hline R34. Enfermedad de un ser querido & 17 & 21.3 & $\begin{array}{l}\text { R29. Muerte de algún amigo/a o } \\
\text { persona querida }\end{array}$ & 8 & 10.0 \\
\hline R13. Hablar en público & 16 & 20.0 & R41. Exceso de trabajo & 6 & 7.5 \\
\hline $\begin{array}{l}\text { R16. Preocupación por la } \\
\text { puntualidad }\end{array}$ & 16 & 20.0 & $\begin{array}{l}\text { R42. Excesiva responsabilidad } \\
\text { laboral }\end{array}$ & 5 & 6.3 \\
\hline
\end{tabular}

Fuente: Elaboración propia a partir de la base de datos 
Resulta interesante notar que las mujeres no reportaron en ninguna de las categorías elementos asociados a las relaciones de pareja o sobre la maternidad. Si bien la comparación por género en los eventos del pasado no fue significativa, al menos tres hombres han experimentado estrés elevado por una ruptura con su pareja o la separación de la misma, de igual forma dos hombres se han estresado por la presencia de un embarazo no deseado.

Enseguida se discutirán los resultados de estas tres categorías, pues resultaron consistentes en que existe un único evento estresante altamente significativo para ambos géneros.

\section{CONCLUSIONES}

La enseñanza de la medicina es consonante con la tendencia del mercado laboral actual que ha determinado como necesidad la adquisición de conocimientos, habilidades y actitudes en la menor cantidad de tiempo. Dicha situación les impone a los futuros profesionistas de la medicina el ingreso a dinámicas de conocimiento mucho más competitivas y demandantes, que en muchos casos vulnera su calidad de vida por el incremento de las expectativas sociales y de una competencia contra una mayor cantidad de personas que desean ingresar a cada vez menos espacios laborales.

En el caso de México y de acuerdo con cifras de 2018 expuestas por Narro (2018) la carrera de medicina ha incrementado su matrícula, pues de 90000 alumnos que se registraron en 2010 se tienen 133000 alumnos en la actualidad. Por otro lado, la alta especialización requerida para ejercer en práctica hospitalaria tiene limitado su acceso, dado que la demanda para la realización de una residencia es alta, tan sólo en 2017 se registraron en el Examen Nacional de Residencias Médicas (ENARM) 38600 aspirantes y sólo se abrieron ocho mil 263 plazas, es decir, sólo un $21.40 \%$ de aspirantes obtuvo un lugar. Estas cifras resultan claras, cada vez existen más médicos buscando un título universitario y una especialidad, situación que es inversa a la oferta de espacios donde puedan desarrollarse.

Ahora bien, esta investigación se centró en estudiar los eventos estresantes de los estudiantes que estaban cursando el primer año de la carrera de medicina en una IES particular, misma que oferta el plan de estudios de la Universidad Nacional 
Autónoma de México (UNAM), cabe mencionar que este plan en su primera fase imparte algunas de las asignaturas con los índices más altos de no aprobación. El Informe de Labores 2008-2015 de la Facultad de Medicina de la UNAM indica que los índices de no aprobación se presentan en las asignaturas de: Anatomía (54.2\%), Bioquímica y Biología Molecular (41.6\%) y Embriología Humana (35\%) que de hecho son parte de los contenidos que se cursan de manera anual en el primer año de este plan de estudios (Graue, 2015) y que resultan consonantes con el momento en que se aplicó el instrumento para este caso de análisis, que fue justo en la segunda etapa semestral de dichos contenidos, en la que una vez que se concluye se define qué alumnos pueden avanzar en su formación, quiénes tendrán que rezagarse y también aquellos que deberán salir del programa académico.

Teniendo en consideración estos datos, resulta comprensible el por qué estos estudiantes tienden a aumentar su percepción de estrés durante los exámenes en esta época en particular, pues el término del primer año es un evento crucial en la formación profesional. Las investigadoras identifican aquí un área de oportunidad para monitorear de manera generacional a estos alumnos y notar su evolución durante toda la carrera.

Aunado a ello, en este trabajo se partió de la idea de que toda persona se encuentra expuesta a vivir situaciones estresantes a lo largo de su vida y en diversos aspectos. En este trabajo, los estudiantes de medicina no se consideraron como sujetos aislados de otros factores que pudieran aumentar la predisposición a experimentar un mayor número de acontecimientos estresantes, al percibir a éstos con mayor intensidad e incluso a recordarlos con mayor frecuencia independientemente del tiempo que haya transcurrido desde que fueron vividos; es por ello que resultó interesante mencionar la frecuencia de respuesta en todos los reactivos con frecuencias significativas, resaltaron aquellos relacionados a aspectos personales y familiares.

En el caso de las comparaciones, se pudo comprobar estadísticamente que las mujeres presentaron medias más altas que los hombres en vigencia e intensidad actual, esto se traduce en que estas estudiantes han experimentado sucesos que les siguen afectando y que además son valorados con un mayor puntaje.

Al respecto de la cuestión de sexo, el visibilizar las diferencias puede apuntar a atender este fenómeno considerando en cuenta las características contextuales; 
en este estudio las mujeres reportaron eventos estresantes relacionados a factores familiares y sociales; como el estilo de vida y amistades. Esto podría marcar nichos de investigaciones futuras al considerar el amplio espectro en el que se mueven las mujeres y a las demandas que frecuentemente deben de responder independientemente de su formación profesional. Autores como Cortés-Flores et al. (2005) indican que en caso de residencias quirúrgicas, la mujer tiene que considerar obstáculos como la discriminación de género, la conciliación entre embarazo y familia, la inequidad salarial y el hostigamiento sexual. Nuevamente apuntamos aquí a profundizar en estudios longitudinales.

Finalmente, el estudio pretende establecer un diferenciador de monitoreo en el bienestar psicológico de la comunidad estudiantil de esta institución que se encuentra incorporada a la Universidad Nacional Autónoma de México (UNAM), misma que en su perfil profesional sugiere que sus estudiantes mantengan su equilibrio emocional, capacidad para el trabajo durante periodos continuos bajo presión, organizar tiempos para el estudio y otras actividades, salud física y mental compatible con el desarrollo de la carrera; es decir, se necesitan estudiantes que sean capaces de regular los acontecimientos estresantes que puedan experimentar a lo largo de su vida académica y personal.

A manera de conclusión, en este trabajo se exploraron los eventos estresantes en alumnos de primer año de un programa académico altamente demandante, se realizó una distinción por sexo y se encontraron diferencias entre las apreciaciones que se hacen de estos elementos, el trabajo realizado sugiere la importancia de realizar un monitoreo periódico, así como el desarrollo de programas para sensibilizar o en su caso entrenar a los estudiantes sobre el manejo del estrés en época de exámenes que fue el evento más significativo para dicha población.

\section{REFERENCIAS}

I American Psychological Association (29 de agosto de 2019) Psychology topics: Stress. Recuperado de https://www.apa.org/topics/stress/index.html

I Becerril, A. (19 de agosto de 2018). Alertan para prevenir suicidio entre médicos residentes. La Jornada. Recuperado de http://www.jornada.com.mx/ultimas/2018/08/19/alertanpara-prevenir-suicidio-entre-medicos-residentes-7561.html 
I Cortes-Flores A. O., Fuentes-Orozco C., López-Ramírez, M. K. L., Velázquez-Ramírez, G. A., Farías-Llamas, O. A., Olivares-Becerra, J. J. y González-Ojeda, A. (2005). Medicina académica y género. La mujer en especialidades quirúrgicas. Gaceta Médica de México. 141(4), jul. /ago. 341-344. Recuperado de http://www.scielo.org.mx/scielo.php?script=sci_ arttext\&pid=S0016-38132005000400016

I Del Pino, M.J. (2012). Género y Estrés. II Jornada mujer y salud "Estrés, género y Salud". Mayo 2012. Recuperado de https://www.sevilla.org/servicios/mujer-igualdad/campanasde-sensibilizacion/28-mayo/historico-campanas-28-de-mayo/pdf-historico-campanas28-de-mayo/ponencia-no-1-2012-ma-jose-del-pino.pdf

I Fernández, J.L. y Mielgo, M. (2001). Escalas de apreciación del Estrés. Madrid: TEA.

I Forbes (21 de diciembre de 2017). Mexicanos los más estresados del mundo por su trabajo. Forbes. Recuperado de https://www.forbes.com.mx/mexicanos-los-mas-estresados-delmundo-por-su-trabajo/

1 García-Viniegras, C. R. y González, I. (2000). La categoría de bienestar psicológico. Su relación con otras categorías sociales. Revista Cubana Medicina General. 16 (6), 586-92. Recuperado de http://scielo.sld.cu/scielo.php?script=sci_ arttext\&pid=S0864-21252000000600010

I Graue, E. (2015) Informe 2012-2015. Facultad de Medicina. Universidad Nacional Autónoma de México. Recuperado de http://www.facmed.unam.mx/_documentos/informe/2015/ inf_2k12_2k15.pdf

I Ministerio de Educación, Cultura y Deporte (2015). Programa para la evaluación Internacional de los Alumnos. El bienestar de los Estudiantes. Ministerio de Educación, Cultura y Deporte. Recuperado de https://www.mecd.gob.es/dctm/inee/internacional/ pisa-2015/informebienestar042517.pdf?documentld=0901e72b8249f043

I Narro, J. R. (2018). Los desafíos de la educación médica en la era de la complejidad. Asociación Mexicana de Facultades y Escuelas de Medicina A.C. (AMFEM). Recuperado de https://www.gob.mx/salud/prensa/239-se-incrementa-en-43-mil-el-numero-deestudiantes-de-medicina-en-los-ultimos-8-anos?idiom=es

I Rodríguez, M. del R., Sanmiguel, M.F., Muñoz, A. y Rodríguez, C.E. (2014). El estrés en estudiantes de medicina al inicio y final de su formación académica. Revista lberoamericana de Educación; 66:105-122. Recuperado de https://rieoei.org/RIE/article/view/381 
Envío a dictamen: 5 de agosto de 2019

Reenvío: 28 de agosto de 2019

Aprobación: 04 de septiembre de 2019

Miriam Sánchez Angeles. Escuela de Medicina. Grupo Educativo Siglo XXI. Zinacantepec, Estado de México. Departamento de Evaluación. Doctora en Ciencias con Énfasis en Educación.

Katya Esquivel Cañas. Campus Universitario Siglo XXI. Zinacantepec, Estado de México. Licenciada en Psicología. 